\title{
Identification $B C L 6$ and miR-30 family associating with Ibrutinib resistance in activated B-cell-like diffuse large B-cell lymphoma
}

\author{
Jiazheng $\mathrm{Li}^{1} \cdot$ Yan Huang ${ }^{1} \cdot$ Yun Zhang ${ }^{1}$ Jingjing Wen ${ }^{1} \cdot$ Yanxin Chen ${ }^{1} \cdot$ Lingyan Wang $^{1} \cdot$ Peifang Jiang ${ }^{1}$. \\ Jianda $\mathrm{Hu}^{1}{ }^{10}$
}

Received: 9 October 2020 / Accepted: 23 January 2021 / Published online: 25 February 2021

(c) The Author(s) 2021

\begin{abstract}
Ibrutinib has clear efficacy for activated B-cell-like diffuse large B cell lymphoma (ABC-DLBCL) in previous clinical researches. However, the resistance of Ibrutinib has limited its therapeutic benefit and the potential mechanism remains unclear. This study was aimed to identify potential candidate genes and miRNA targets to overcome Ibrutinib resistance in ABC-DLBCL. First, two expression profiles were downloaded from the GEO database, which used to identify the DEGs related to Ibrutinib resistance in ABC-DLBCL cell lines by GEO2R analysis separately. And the common DEGs were obtained though Venn diagram. Then Gene ontology (GO) and pathway enrichment analysis were conducted by DAVID database. From STRING database, BCL6, IL10, IL2RB, IRF4, CD80, PRDMI and GZMB were determined to be the hub genes by protein-protein interaction (PPI) network. Through miRNA-mRNA targeting network, we found that $B C L 6, I R F 4$, $C D 80$, and PRDM1 were common target genes of miR-30 family. The cBioPortal database showed that $B C L 6$ had the highest level of genetic alterations among DLBCL. In addition, another expression profile from GEO database showed that BCL6 was significantly high expression in no responsive patients after Ibrutinib treatment, and the receiver operating characteristic (ROC) curve which was used to evaluate the relationship between BCL6 expression and its effect was 0.67. MTT assay showed that treatment with FX1 (a BCL6 inhibitor) can enhance the sensitivity of Ibrutinib in C481S BTK HBL-1 cells. The results suggested that $B C L 6$ and miR-30 family maybe associate with Ibrutinib resistance in ABC-DLBCL.
\end{abstract}

Keywords Activated B-cell-like diffuse large B cell lymphoma $\cdot$ Ibrutinib resistance $\cdot$ Bioinformatic analysis $\cdot B C L 6 \cdot \mathrm{miR}$ 30 family

\section{Introduction}

Diffuse large B cell lymphoma (DLBCL) were classified as germinal center B-cell-like (GCB) and activated B-cell-like $(\mathrm{ABC})$ DLBCL with cell-of-origin (COO) $[1,2]$. And the $A B C$ subtype presents poor prognosis [3]. Although targeted therapy drugs improved the prognosis of DLBCL patients. However, about $40 \%$ patients of DLBCL could not benefit

Jiazheng Li, Yan Huang and Yun Zhang contributed equally as co-first authors.

Jianda $\mathrm{Hu}$

drjiandahu@163.com

1 Fujian Provincial Key Laboratory of Hematology, Fujian Institute of Hematology, Fujian Medical University Union Hospital, 29 Xinquan Road, Fuzhou 350001, China from first-line therapy, and drug resistance is a leading cause of it [4].

Drug resistance relates to various mechanisms, such as gene-driven, pathway mediated. TBLIXR1, IRF4, TP53, FOXO1, KMT2C (MLL3), CCND3, NFKBIZ, and STAT6, were potential candidate targets to overcome drug resistance in DLBCL [5, 6]. In addition, increasing evidences have revealed miRNAs negatively regulated expression of their target genes and abnormally expressed in many tumors, including DLBCL [7, 8]. A meta-analysis suggests that DLBCL patients with abnormal expression of miR-155, miR-17/92 clusters, miR-21, miR-224, or miR-146b-5p are associated with worse outcome and higher risk of drug resistance [9]. Currently, more and more DLBCL related genes and signaling pathways have been identified, and a number of targeted therapeutic drugs have recently been introduced, such as Ibrutinib. 
Ibrutinib, a small molecule inhibitor of Brutons tyrosine kinase (BTK), was approved to use for several B-cell malignancies by the United States Food and Drug Administration (FDA) in 2013 [10]. The curative effect of Ibrutinib monotherapy on rel/ref ABC DLBCL is significantly better than that of GCB subtype [11]. However, resistance to ibrutinib limits its effectiveness, and the underling mechanisms are still not clear. Bioinformatics analysis is used to analyze data of high-throughput sequencing, which helps us to study potential molecular mechanisms of drug resistance. The present study generated differentially expressed genes (DEGs) to identified the core gene among their regulatory relations and miRNA targets associated with Ibrutinib resistance in ABC-DLBCL.

\section{Materials and methods}

\section{Microarray data}

The three microarray expression profile datasets (GSE138126 、GSE93984 and GSE93985) were obtained from the gene expression omnibus (GEO, http://www. ncbi.nlm.nih.gov/geo/) database [12]. GSE138126 was determined by GPL13497 Agilent-026652 Whole Human Genome Microarray $4 \times 44$ K (Submission date: Sep 29, 2019, Last update date: Nov 03, 2019), a total of 6 Ibrutinibresistant and 6 sensitive samples, including HBL-1 and OCILY10 ABC DLBCL cell lines. And GSE93985 was based on the comparison between sensitive TMD8 ABC DLBCL and its Ibrutinib-resistant cell lines. It was performed by GPL17586.0 Affymetrix Human Transcriptome Array 2.0 (Submission date: Jan 24, 2017, Last update date: Oct 29, 2018). The GSE93984 was detected by GPL570 U133 plus 2.0 arrays, which included Ibrutinib pretreated tumor biopsy samples from ABC-DLBCL patients.

\section{DEGs screening}

The DEGs between Ibrutinib sensitive and resistant cell lines were obtained by GEO2R analysis with cutoff values of $P$ value $<0.05$ and $\mid \log _{2} \mathrm{FCl}>1$ [13], a web application based on $\mathrm{R}$ software in GEO database. Then we used limma package of $\mathrm{R}$ software to constructed Volcano maps showing the DEGs and Draw Venn Diagram website (http://bioinforma tics.psb.ugent.be/webtools/Venn/) to get the overlapped DEGs. The overlapped DEGs were considered to be associated with Ibrutinib resistance.

\section{Gene ontology (GO) and pathway analysis}

GO analysis was carried out to analyze different functions of Ibrutinib resistance-related DEGs including biological process (BP), cellular component (CC), molecular function (MF) category. Pathway enrichment analysis was carried out with Kyoto Encyclopedia of Genes and Genomes (KEGG) and Reactome database. In this work, GO terms and pathway analysis were both performed by Annotation, Visualization and Integrated Discovery (DAVID, http://david.abcc.ncifc rf.gov/) (vision 6.8) [14] database with $P$ value $<0.05$ as the threshold value.

\section{Integration of protein-protein interaction (PPI) network}

Search Tool for the Retrieval of Interacting Genes (STRING, http://string-db.org) (vision 11.0) [15] database was used to generate PPI network to show the association among DEGs. Further, PPI network was visualized through cytoscape (vision 3.7.2) software [16], then the top DEGs with Maximal Clique Centrality (MCC) score $>10,000$ were regarded as hub genes by CytoHubba plugin.

\section{Exploring genetic alterations of hub genes and predicting hub gene-related miRNAs}

The cBioPortal database (http://www.cbioportal.org/) [17] was used to explore genetic alterations of hub genes. The miRNAs who target hub genes were predicted by miRDB database (http://mirwalk.umm.uni-heidelberg.de) [18] and the miRNA-mRNA interaction was constructed by Cytoscape (vision 3.7.2) software.

\section{BCL6 expression in ABC-DLBCL patients with different outcome after Ibrutinib treatment}

In the GSE93984 expression profile, 17 ABC-DLBCL patients were classified into no responsive (stable disease + progression disease, SD + PD) group, and $11 \mathrm{ABC}$ DLBCL patients were responsive (complete response + partial response, $\mathrm{CR}+\mathrm{PR}$ ) group after Ibrutinib treatment. We used the GraphPad Prism Software 7.0 to visualize the relative expression of $B C L 6$ between above two groups, and the ROC R package was performed to operate ROC curves.

\section{Cell culture}

C481S BTK HBL-1 cells, the BTK C481S mutant induced resistance to Ibrutinib, were a gift from Prof. Zhu J (Key laboratory of Carcinogenesis and Translational Research, Department of Lymphoma, Peking University Cancer Hospital \& Institute). C481S BTK HBL-1 cells were cultured in RPMI-1640 medium (BasalMedia, China), 10\% fetal bovine serum (FBS, Gibco, UT) and $0.05 \mathrm{mM}$ 2-mercaptoethanol (Sigma, USA) at $37{ }^{\circ} \mathrm{C}$ with $5 \% \mathrm{CO}_{2}$ with in a humidified incubator (Thermo, USA). 


\section{Ibrutinib sensitivity assay}

The effect of Ibrutinib or combination with FX1 (a BCL6 inhibitor) on C481S BTK HBL-1 cells was detected by 3-(4,5-dimethylthiazol2-yl)-2,5 diphenyltetra-zolium bromide (MTT, Sigma, MO, USA) assay in vitro. C481S BTK HBL- 1 cells were seeded at a density of $4 \times 10^{4} /$ well and incubated with different concentrations of Ibrutinib $(0,0.5$, $1,2,4$, and $8 \mu \mathrm{M}$ ) with/without $17.5 \mu \mathrm{M} \mathrm{FX} 1$ at $37{ }^{\circ} \mathrm{C}$ in a $5 \% \mathrm{CO}_{2}$ incubator for $48 \mathrm{~h}$. Then cells were incubated with $20 \mu \mathrm{L}$ MTT $(5 \mathrm{mg} / \mathrm{mL})$ in the last four hours of the experiment. Optical density (OD) value was measured at the wave length of $490 \mathrm{~nm}$ and $630 \mathrm{~nm}$ by an Elx808 Absorbance Microplate spectrophotometer (BioTek, UT).

\section{Statistics analysis}

Each experiment was performed in triplicate. Measurement data were expressed as mean \pm standard deviations and unpaired $t$ test, processed by SPSS statistics software 25.0 or GraphPad Prism Software 7.0, where $P<0.05$ means significant statistical differences.

\section{Results}

\section{Identification of DEGs between Ibrutinib sensitive and resistant cell lines in ABC-DLBCL}

The two mRNA expression profiles, GSE138126 and GSE93985, were obtained from the GEO database including 3 ABC-DLBCL cell lines (HBL-1, OCI-LY10, TMD8), as shown in Table 1. We obtained 6191, 7063, 2531 DEGs from above 3 cell lines, respectively, with $P<0.05$ and $\mid \log _{2} \mathrm{FCl} \geq 1$ as cutoff values by GEO2R analysis. Volcano plot of the DEGs between Ibrutinib sensitive and resistant cell lines were generated (Fig. 1a-c). A total of 671 common DEGs were identified from above 3 cell lines, and 236 of 671 common DEGs showed concordant expression changes, among them, 136 downregulated and 100 upregulated (Fig. 1d, e). Therefore, above 236 common DEGs would be used for subsequent analysis.

\section{GO analysis and pathway analysis}

We performed GO analysis to analyze the functional role of resistance-related DEGs using DAVID database (vision 6.8). The top 5 GO terms were selected to display according to count (numbers of related gene). Biological process indicated that upregulated DEGs were correlated with transcription (Fig. 2a) while downregulated DEGs were signal transduction and negative regulation of apoptotic process (Fig. 2d). And both the enrichment in cellular components mainly related to nucleus and cytoplasm (Fig. 2b, e). Under the category of molecular function, upregulated DEGs were significantly enriched in DNA binding and chromatin binding (Fig. 2c), whereas downregulated DEGs were mainly enriched in protein binding (Fig. 2f).

KEGG Pathway and Reactome database were performed to learn Ibrutinib resistance-related signaling pathway in ABC-DLBCL. The Table 2 and Table 3 listed the downregulated and upregulated DEGs significantly enriched pathway $(P<0.05)$, respectively. The common signaling pathway was cell cycle pathway in upregulated DEGs while cytokinesbased signaling pathways in downregulated DEGs.

\section{PPI network and hub genes identification}

String database and Cytoscape (vision 3.7.2) software were used to study and visualize the correlation among the DEGs at the protein level. The Ibrutinib resistance-related PPI network included 228 nodes and 430 edges with PPI enrichment $(P$ value $<1.0 \mathrm{e}-16)$ (Fig. 3a). The top 10 DEGs based on MCC score in above PPI network were identified according to cytoHubba plugin in Cytoscape software as in Fig. 3b. With the threshold of MCC score $>10,000$, a total of seven genes were considered hub genes: BCL6, IL10, IL2RB, IRF4, CD80, PRDM1, GZMB (Fig. 3c). And BCL6 got the highest score so that $B C L 6$ may be the most important gene among them.

\section{Construction of miRNA-mRNA interaction}

MiRDB database was used to analyze miRNA associated with hub genes which might affect Ibrutinib resistance in DLBCL. Every predicted miRNA in miRDB database has a prediction score so that we defined $>70$ as the threshold level. However, we could not find miRNA that targets

Table 1 Information of the two microarray expression profiles

\begin{tabular}{llll}
\hline GEO ID & Platform & Cell line & Samples \\
\hline GSE138126 & GPL13497 Agilent-026652 Whole Human Genome Microarray 4×44 K & HBL-1 & 3 Ibrutinib-resistant/3 parental cell lines \\
GSE138126 & GPL13497 Agilent-026652 Whole Human Genome Microarray 4×44 K & OCI-LY10 & 3 Ibrutinib-resistant/3 parental cell lines \\
GSE93985 & GPL17586[HTA-2_0] Affymetrix Human Transcriptome Array 2.0 & TMD8 & 2 Ibrutinib-resistant/2 parental cell lines \\
\hline
\end{tabular}




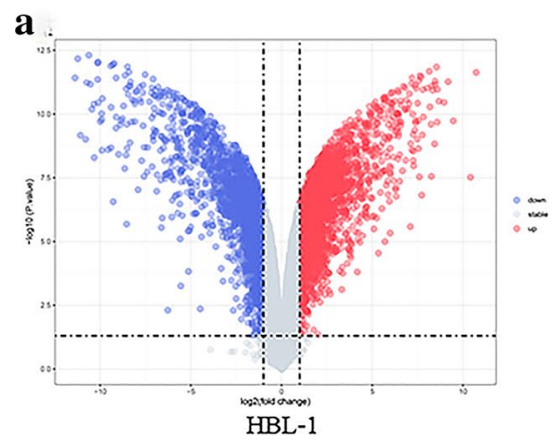

b

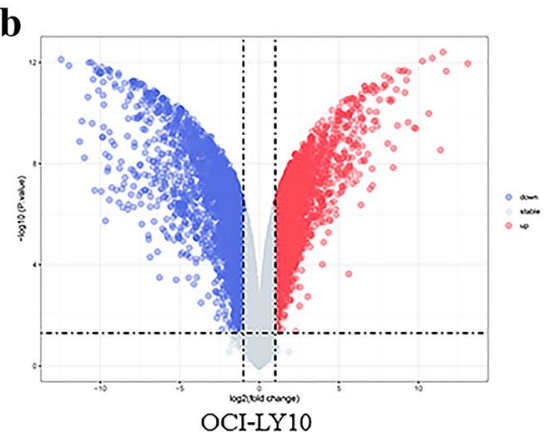

c

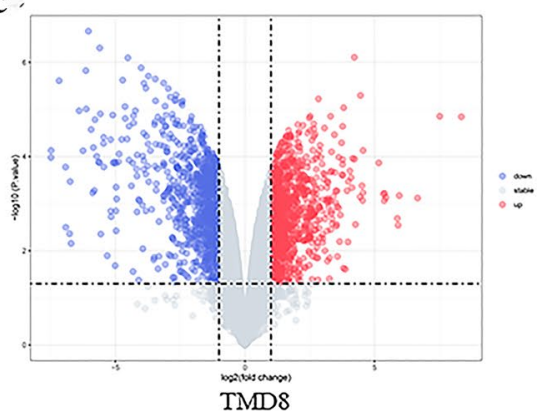

d

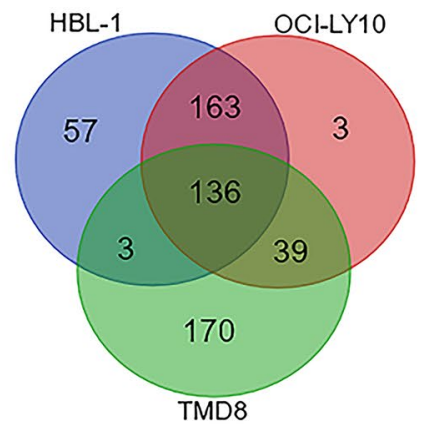

Fig. 1 Identification of DEGs between Ibrutinib-resistant cell lines and sensitive cell lines in ABC-DLBCL. Volcano plot of a HBL-1, b OCI-LY10 and c TMD8. Blue plots represent downregulated genes, while red plots represent upregulated genes. Identification of the oncordant expression changes d downregulated e upregulated DEGs

GZMB. Next, Cytoscape software was used to generate miRNA-mRNA interaction among 6 hub genes (Fig. 4). We found that hsa-miR-30a-5p, hsa-miR-30b-5p, hsa-miR30c-5p, hsa-miR-30d-5p, and hsa-miR-30e-5p targeted for BCL6, PMDR1, CD80, and IRF4 simultaneously. And above miRNAs belong to miRNA-30 family members.

\section{Genetic alteration of hub genes}

We used cBioportal database to explore the genetic alterations of hub genes including 1295 samples in five DLBCL datasets (DFCI, Nat Med 2018, BCGSC, Blood 2013, Broad, PNAS 2012, Duke, Cell 2017, TCGA, PanCancer Atlas) (Fig. 5). Genetic alteration of IL2RB was not found while genetic alterations of the remaining six hub genes included mutation, fusion, multiple alterations, amplification, and deep deletion. Mutation was the most common genetic alterations among these hub genes. Significantly, we found that $B C L 6$ showed the highest level of genetic alterations among hub genes. According to the result of PPI network and genetic alterations, we thought the BCL6 may play the most important role among these hub genes. Therefore, we validated the $B C L 6$ in following analysis and experiment. e

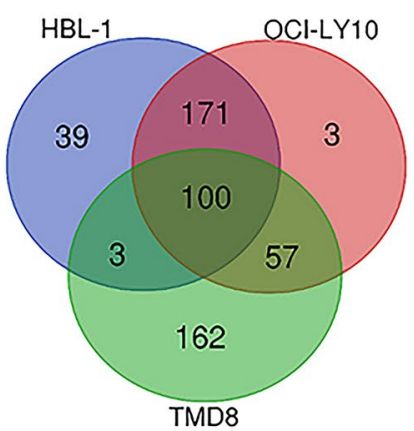

from three cell lines were performed by Draw Venn Diagram website (http://bioinformatics.psb.ugent.be/webtools/Venn/). Blue circles indicate HBL-1 cell line, red circles indicate OCI-LY10 cell line, green circles indicate TMD8 cell line

\section{Validation of $B C L 6$}

We got the $B C L 6$ expression in Ibrutinib pretreated tumor biopsy samples of ABC-DLBCL patients from GSE93984 dataset. The $B C L 6$ was significantly highly expressed in no responsive than responsive patients (Fig. 6a). The ROC analysis showed that $B C L 6$ expression can evaluate Ibrutinib effect with an area under ROC curve (AUC) of 0.67 (Fig. 6b). It suggested well confidence of high expression of $B C L 6$ in no responsive patients after Ibrutinib treatment. We used MTT assay to determine the effect of Ibrutinib with/ without FX1 (a BCL6 inhibitor) on C481S BTK HBL-1 cells. The inhibition rate of C481S BTK HBL-1 cells increased with increasing concentrations of Ibrutinib (Fig. 6c). The $\mathrm{IC}_{50}$ value of using Ibrutinib alone was $1.951 \pm 0.247 \mu \mathrm{M}$, while combining FX1 was $0.800 \pm 0.137 \mu \mathrm{M}$ (Fig. 6d). Thus, FX1 can enhance Ibrutinib sensitivity in C481S BTK HBL-1 cells, which indicated that BCL6 can decrease Ibrutinibinduced apoptosis in C481S BTK HBL-1 cells. 
a

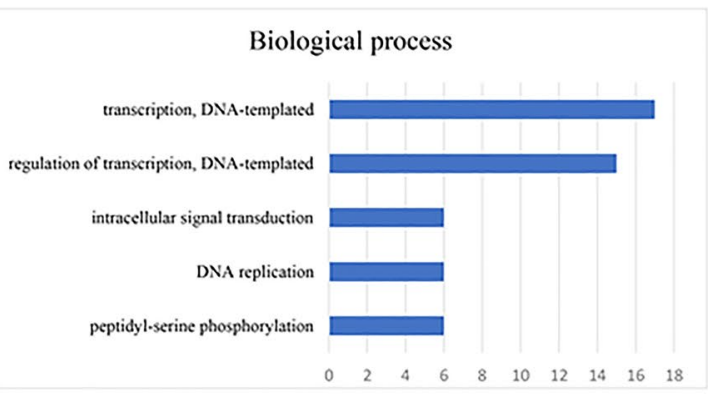

b

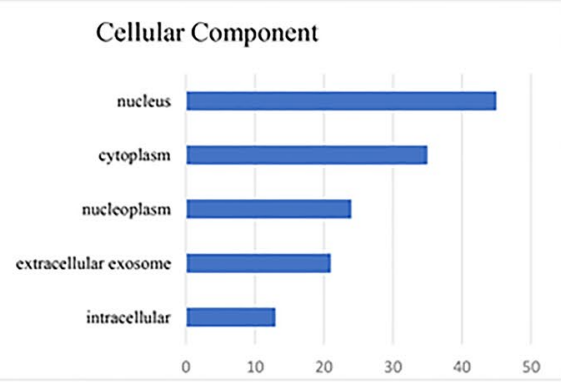

c

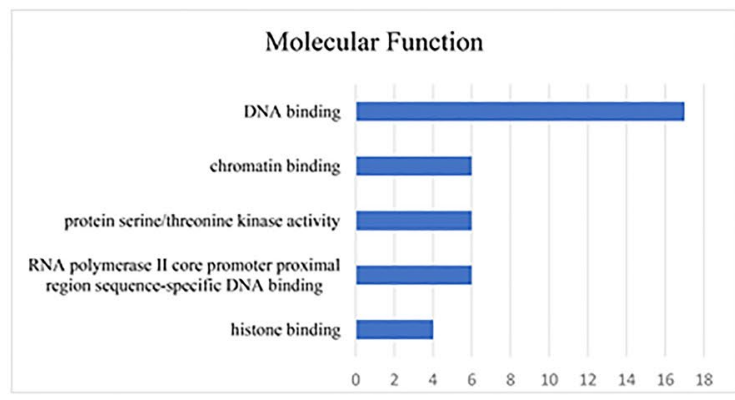

Fig. 2 GO analysis of DEGs. Enrichment of a biological process, b cellular components, and c molecular function for upregulated DEGs. Enrichment of $\mathbf{d}$ biological process, e cellular components, d

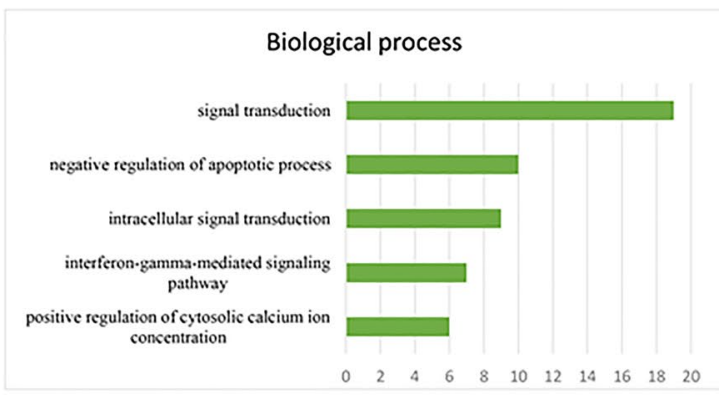

e

Cellular Component

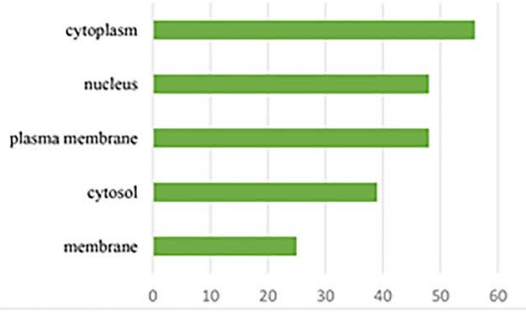

f

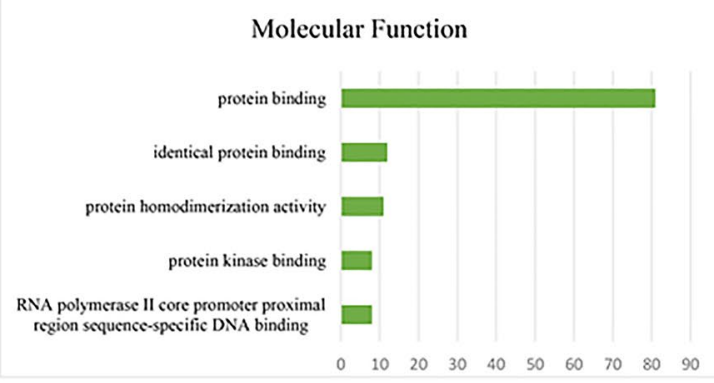

Table 2 The significant pathway enrichment analysis of upregulated DEGs

\begin{tabular}{llll}
\hline Category & Term & $P$ value & Genes \\
\hline KEGG & hsa04110: cell cycle & $1.78 \mathrm{E}-02$ & HDAC1, CDKN2C, CDC25A, ATM \\
KEGG & hsa04910: insulin signaling pathway & $2.36 \mathrm{E}-02$ & PPP1R3E, PRKAR2A, PRKCI, PIK3R3 \\
KEGG & hsa04390: hippo signaling pathway & $2.97 \mathrm{E}-02$ & PPP2R1B, TEAD4, PRKCI, LEF1 \\
KEGG & hsa05202: transcriptional misregulation in cancer & $3.84 \mathrm{E}-02$ & HDAC1, CDKN2C, BCL6, ATM \\
REACTOME & R-HSA-113510: E2F mediated regulation of DNA replication & $2.85 \mathrm{E}-03$ & DHFR, RRM2, CDC25A \\
REACTOME & R-HSA-69205: G1/S-specific transcription & $2.85 \mathrm{E}-03$ & DHFR, RRM2, CDC25A \\
REACTOME & R-HSA-1538133: G0 and early G1 & $6.14 \mathrm{E}-03$ & RBBP4, HDAC1, CDC25A \\
\hline
\end{tabular}

\section{Discussion}

Even though patients of DLBCL respond sensitively to firstline treatment, approximately $40 \%$ of patients still could not benefit from it [4]. Ibrutinib is an inhibitor of BTK, showed obvious efficacy in rel/ref DLBCL, especially ABC subtype and $\mathbf{f}$ molecular function for downregulated DEGs. DEGs functional enrichment was analyzed using GO analysis on DAVID database (vision 6.8) 
Table 3 The significant pathway enrichment analysis of downregulated DEGs

\begin{tabular}{|c|c|c|c|}
\hline Pathway & Term & $P$ value & Genes \\
\hline KEGG & hsa05330: allograft rejection & 4.19E-05 & $\begin{array}{l}\text { CD80, GZMB, HLA-DPA1, HLA-DPB1, HLA-DOA, } \\
\text { IL10 }\end{array}$ \\
\hline KEGG & hsa04672: intestinal immune network for $\operatorname{IgA}$ production & $1.36 \mathrm{E}-04$ & $\begin{array}{l}\text { CD80, CCR10, HLA-DPA1, HLA-DPB1, HLA-DOA, } \\
\text { IL10 }\end{array}$ \\
\hline KEGG & hsa05145: toxoplasmosis & $1.64 \mathrm{E}-04$ & $\begin{array}{l}\text { CIITA, HLA-DPA1, JAK2, HLA-DPB1, HLA-DOA, } \\
\text { IL10, AKT3, STAT3 }\end{array}$ \\
\hline KEGG & hsa05320: autoimmune thyroid disease & $2.20 \mathrm{E}-04$ & $\begin{array}{l}\text { CD80, GZMB, HLA-DPA1, HLA-DPB1, HLA-DOA, } \\
\text { IL10 }\end{array}$ \\
\hline KEGG & hsa05332: graft-versus-host disease & $3.99 \mathrm{E}-04$ & CD80, GZMB, HLA-DPA1, HLA-DPB1, HLA-DOA \\
\hline KEGG & hsa04940: type I diabetes mellitus & $1.01 \mathrm{E}-03$ & CD80, GZMB, HLA-DPA1, HLA-DPB1, HLA-DOA \\
\hline KEGG & hsa05152: tuberculosis & $2.79 \mathrm{E}-03$ & $\begin{array}{l}\text { CIITA, HLA-DPA1, JAK2, HLA-DPB1, HLA-DOA, } \\
\text { IL10, AKT3, CD74 }\end{array}$ \\
\hline KEGG & hsa05416: viral myocarditis & $3.17 \mathrm{E}-03$ & CD55, CD80, HLA-DPA1, HLA-DPB1, HLA-DOA \\
\hline KEGG & hsa05310: asthma & $3.94 \mathrm{E}-03$ & HLA-DPA1, HLA-DPB1, HLA-DOA, IL10 \\
\hline KEGG & hsa05321: inflammatory bowel disease (IBD) & $4.82 \mathrm{E}-03$ & HLA-DPA1, HLA-DPB1, HLA-DOA, IL10, STAT3 \\
\hline KEGG & hsa04920: adipocytokine signaling pathway & $6.62 \mathrm{E}-03$ & CD36, SOCS3, JAK2, AKT3, STAT3 \\
\hline KEGG & hsa05140: leishmaniasis & $6.96 \mathrm{E}-03$ & HLA-DPA1, JAK2, HLA-DPB1, HLA-DOA, IL10 \\
\hline KEGG & hsa04612: antigen processing and presentation & $8.83 \mathrm{E}-03$ & CIITA, HLA-DPA1, HLA-DPB1, HLA-DOA, CD74 \\
\hline KEGG & hsa05164: influenza A & $1.08 \mathrm{E}-02$ & $\begin{array}{l}\text { CIITA, SOCS3, HLA-DPA1, JAK2, HLA-DPB1, HLA- } \\
\text { DOA, AKT3 }\end{array}$ \\
\hline KEGG & hsa04978: mineral absorption & $1.15 \mathrm{E}-02$ & HMOX1, MT2A, STEAP1, MT1F \\
\hline KEGG & hsa04062: chemokine signaling pathway & $1.46 \mathrm{E}-02$ & LYN, PREX1, HCK, CCR10, JAK2, AKT3, STAT3 \\
\hline KEGG & hsa04630: jak-STAT signaling pathway & $1.95 \mathrm{E}-02$ & IL2RB, SOCS3, JAK2, IL10, AKT3, STAT3 \\
\hline KEGG & hsa05150: staphylococcus aureus infection & $2.00 \mathrm{E}-02$ & HLA-DPA1, HLA-DPB1, HLA-DOA, IL10 \\
\hline KEGG & hsa04145: phagosome & $2.23 \mathrm{E}-02$ & $\begin{array}{l}\text { CD36, TFRC, TUBB6, HLA-DPA1, HLA-DPB1, HLA- } \\
\text { DOA }\end{array}$ \\
\hline KEGG & hsa04931: insulin resistance & $2.86 \mathrm{E}-02$ & CD36, SOCS3, PTPN1, AKT3, STAT3 \\
\hline KEGG & hsa04917: prolactin signaling pathway & $4.06 \mathrm{E}-02$ & SOCS3, JAK2, AKT3, STAT3 \\
\hline KEGG & hsa05169: epstein-Barr virus infection & 4.19E-02 & LYN, HLA-DPA1, HLA-DPB1, AKT3, STAT3 \\
\hline KEGG & hsa05168: herpes simplex infection & $4.65 \mathrm{E}-02$ & $\begin{array}{l}\text { SOCS3, HLA-DPA1, JAK2, HLA-DPB1, HLA-DOA, } \\
\text { CD74 }\end{array}$ \\
\hline REACTOME & R-HSA-877300: interferon gamma signaling & $1.34 \mathrm{E}-05$ & $\begin{array}{l}\text { CIITA, TRIM2, SOCS3, MT2A, HLA-DPA1, JAK2, } \\
\text { IRF4, HLA-DPB1 }\end{array}$ \\
\hline REACTOME & R-HSA-982772: growth hormone receptor signaling & $5.73 \mathrm{E}-05$ & LYN, SOCS3, JAK2, PTPN1, STAT3 \\
\hline REACTOME & R-HSA-2132295: MHC class II antigen presentation & $7.94 \mathrm{E}-04$ & $\begin{array}{l}\text { EHHADH, CTSO, TUBB6, HLA-DPA1, HLA-DPB1, } \\
\text { HLA-DOA, CD74 }\end{array}$ \\
\hline REACTOME & R-HSA-389513: CTLA4 inhibitory signaling & $9.67 \mathrm{E}-04$ & LYN, CD80, PPP2R5C, AKT3 \\
\hline REACTOME & R-HSA-114604: GPVI-mediated activation cascade & $1.59 \mathrm{E}-03$ & IL2RB, GAB2, LYN, JAK2, AKT3 \\
\hline REACTOME & R-HSA-2586552: signaling by Leptin & $4.21 \mathrm{E}-03$ & SOCS3, JAK2, STAT3 \\
\hline REACTOME & R-HSA-1059683: interleukin-6 signaling & $4.21 \mathrm{E}-03$ & SOCS3, JAK2, STAT3 \\
\hline REACTOME & R-HSA-1433557: signaling by SCF-KIT & $4.43 \mathrm{E}-03$ & GAB2, LYN, JAK2, STAT3 \\
\hline REACTOME & R-HSA-877312: regulation of IFNG signaling & $6.84 \mathrm{E}-03$ & SOCS3, JAK2, PTPN1 \\
\hline REACTOME & $\begin{array}{l}\text { R-HSA-392451: G beta:gamma signaling through PI3K- } \\
\text { gamma }\end{array}$ & $9.18 \mathrm{E}-03$ & IL2RB, GAB2, JAK2, AKT3 \\
\hline REACTOME & R-HSA-912526: interleukin receptor SHC signaling & $2.45 \mathrm{E}-02$ & IL2RB, GAB2, JAK2 \\
\hline REACTOME & $\begin{array}{l}\text { R-HSA-202433: generation of second messenger mol- } \\
\text { ecules }\end{array}$ & 4.17E-02 & ENAH, HLA-DPA1, HLA-DPB1 \\
\hline
\end{tabular}

(R-CHOP) improved event-free survival (EFS), progression-free survival (PFS), overall survival (OS) in ABC DLBCL patients [20]. Increasing clinical trials investigate
Ibrutinib curative effect on DLBCL. We knew that Ibrutinib was approved to be used for several B-cell malignancies in FDA in 2013 [10]. However, the emergence of Ibrutinib 


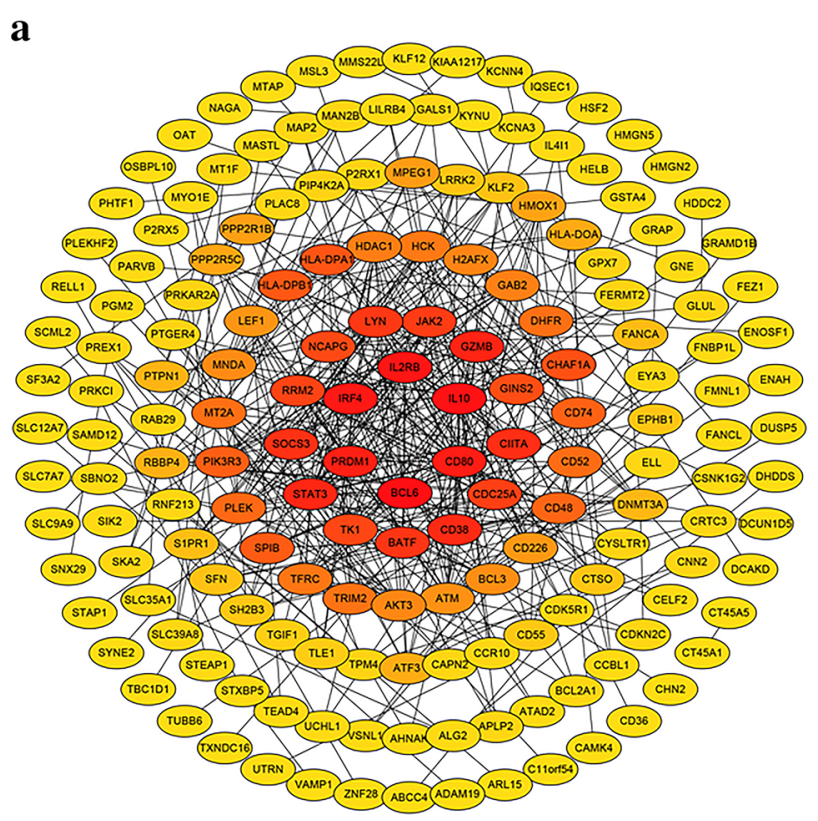

b
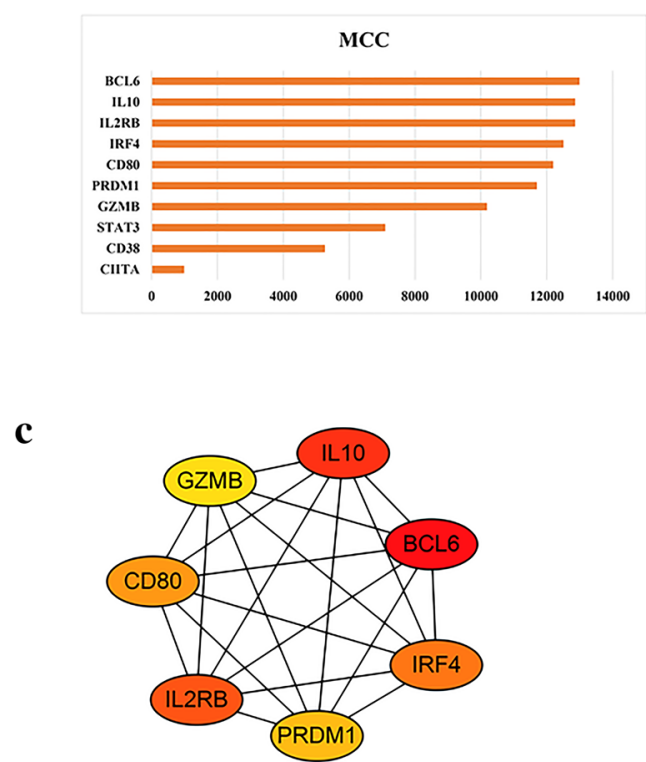

Fig. 3 The PPI network and hub genes identification. a The PPI network of DEGs was visualized by Cytoscape software. b The top 10 DEGs based on MCC algorithm analysis. $\mathbf{c}$ The hub genes were identified with the MCC score cutoff of 10,000
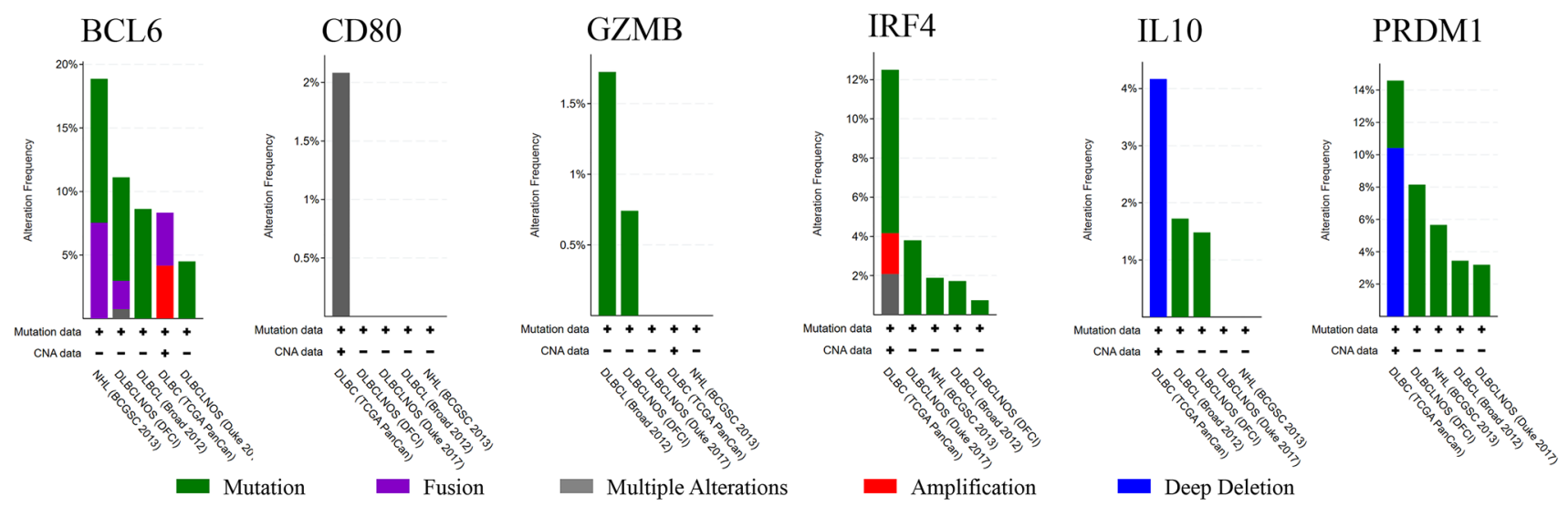

Fig. 4 The histogram of the genetic alteration frequencies of hub genes across five DLBCL datasets (DFCI, Nat Med 2018, BCGSC, Blood 2013, Broad, PNAS 2012, Duke, Cell 2017, TCGA, PanCancer Atlas)

resistance has limited its efficacy [21, 22], so it's important to study the mechanism of Ibrutinib resistance in DLBCL.

In this study, three microarray expression profile datasets were analyzed to further study mechanism of Ibrutinib resistance. First, a total of 237 common DEGs between Ibrutinib sensitive and resistant cell lines were identified through two expression profiles, including 100 upregulated and 137 downregulated genes. Second, GO analysis was performed to study functional roles, including biological process, molecular functions and cellular component. The upregulated DEGs were correlated with transcription and downregulated DEGs were signal transduction and negative regulation of apoptotic process. Third, signal pathway enrichment analysis showed that upregulated DEGs were mainly enriched in cell cycle and downregulated DEGs were mainly enriched in cytokines-based pathways. What is more, PPI network was conducted to illustrate interactions among the DEGs at the protein level, of which, a total of 7 genes-BCL6, IL10, IL2RB, IRF4, CD80, PMDR1, GZMBwere selected as hub genes with the threshold of MCC score $>10,000$. And through predicting miRNA associated with hub genes, we found that miR-30 family may be related to Ibrutinib resistance in ABC-DLBCL. From the cBioportal database, we found that $B C L 6$ showed the highest level of 


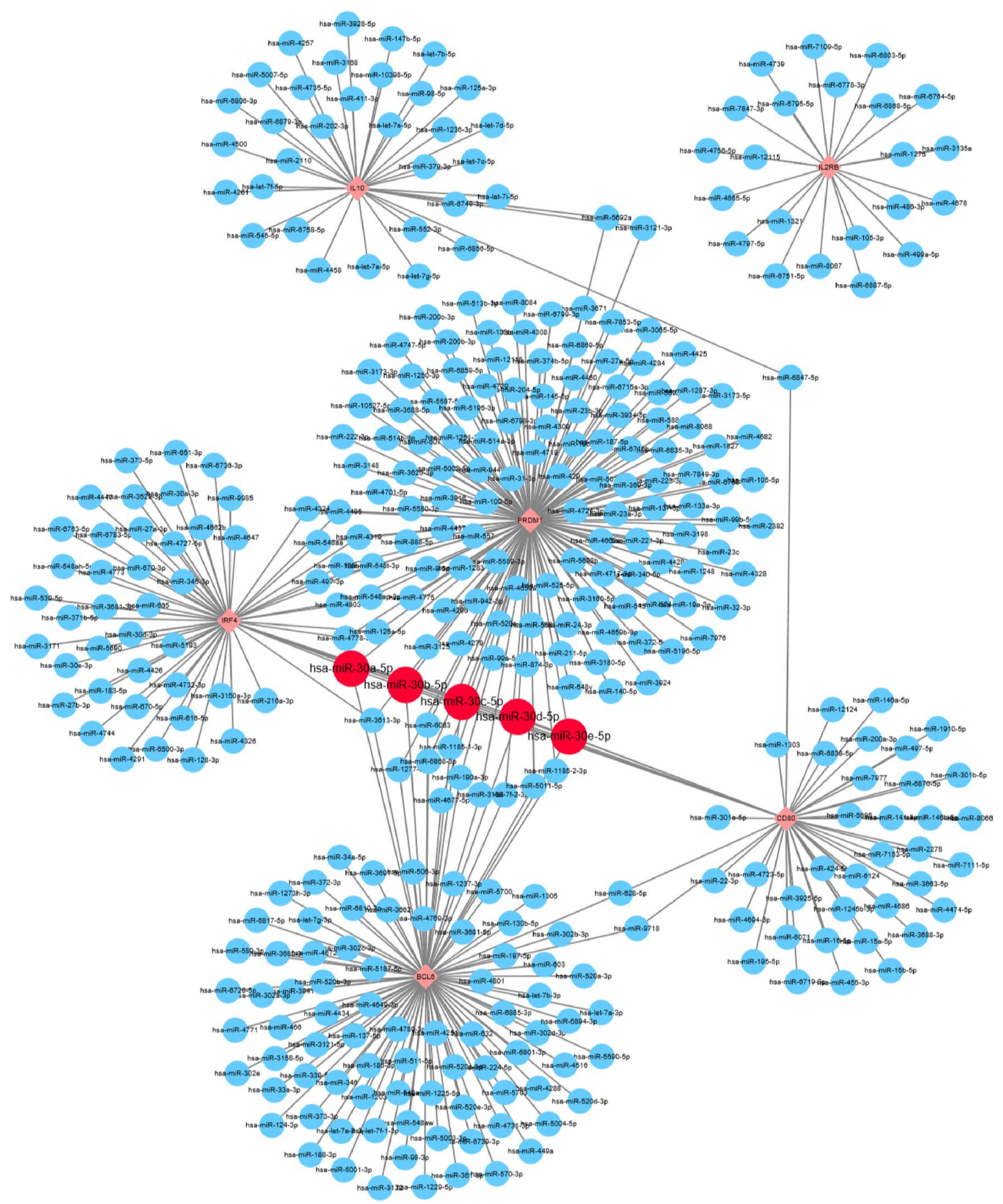

Fig. 5 The construction of miRNA-mRNA interaction of hub genes in ABC-DLBCL. Diamond represents hub gene and circle represents predicted the potential miRNA

genetic alterations among above hub genes. Using another expression profile, we found that $B C L 6$ highly expressed in no responsive $\mathrm{ABC}-\mathrm{DLBCL}$ patients, and the AUC of ROC curve was 0.67 .

$B C L 6$, a transcription repressor, plays an important role of initiation and maintenance of germinal center reactions $[23,24]$, which has been identified as one of predictors of outcome in several cancers, such as DLBCL and B-cell acute lymphoblastic leukemia (B-ALL) [25, 26]. It was reported that $B C L 6$ is associated with tyrosine kinase inhibitors (TKI) resistance in Philadelphia chromosome positive $(\mathrm{Ph}+)$ ALL and chronic myeloid leukemia (CML) cells [27, 28]. And another study showed overexpression of BCL6 inhibited the sensitivity of methotrexate in children with B-ALL by 
Fig. 6 Validation of $B C L 6$. a $B C L 6$ was highly expressed in no responsive $\mathrm{ABC}-\mathrm{DLBCL}$ patients after Ibrutinib treatment. b ROC curve for discriminating no response or response through $B C L 6$ expression. c C481S BTK HBL-1 cells were treated with 0-8 uM Ibrutinib or combination with $17.5 \mathrm{uM}$ FX1. d MTT-based assessment of the $\mathrm{IC}_{50}$ value of Ibrutinib compared with Ibrutinib combining FX1. Values were calculated mean $\pm \mathrm{SD}(n=3$, and $* * P<0.01)$
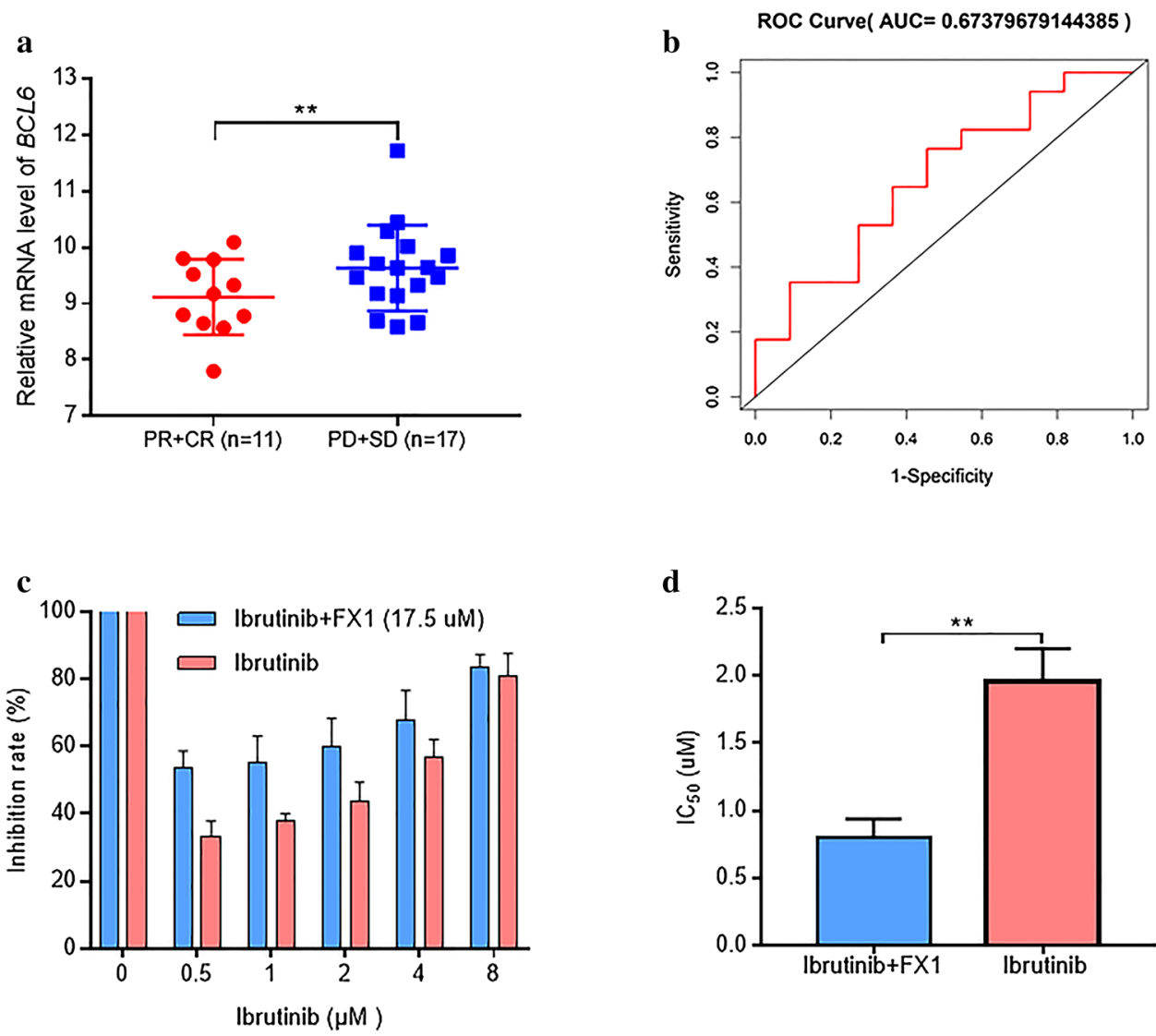

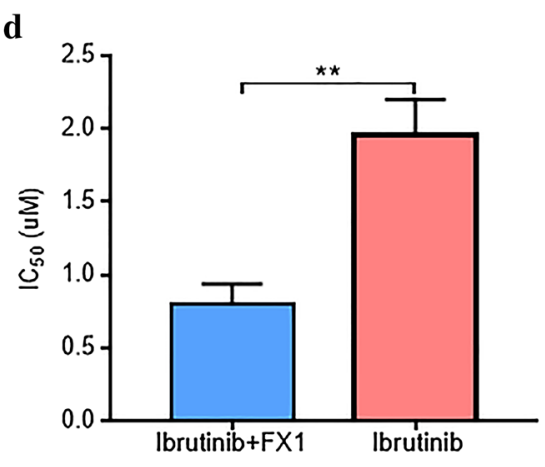

promoting ZEBI expression [29]. What is more, Julie et al. found association between BCL6 overexpression and etoposide resistance in DLBCL cell lines [30]. Cardenas et al. reported that BCL6 expresses in most ABC-DLBCL at a low level [31]. It was interesting that BCL6 was upregulated in Ibrutinib-resistant ABC-DLBCL cell lines in our study. And BCL6 had the highest MCC score in Ibrutinib-resistant PPI network and linked with another hub genes. So using another gene profile validated that BCL6 highly expressed in no responsive ABC-DLBCL patients after Ibrutinib treatment. And in vitro experiment was carried out to validate if BCL6 inhibitor can enhance the sensitivity of Ibrutinib in C481S BTK HBL-1 cells. FX1, a BCL6 inhibitor, destroyed the formation of $B C L 6$ repression complex and suppressed ABC-DLBCL cell lines with $\mathrm{IC}_{50}$ of $35 \mathrm{uM}$ [31]. FX1 used in our study was lower than its $\mathrm{IC}_{50}$, which can increase sensitivity of Ibrutinb in C481S BTK HBL-1 cells. Thus, our finding that BCL6 may be involved in drug resistance is consistent with previous studies. What is more, BCL6 maybe the potential target to improve Ibrutinib sensitivity in C481S BTK HBL-1 cells.

$B C L 6$ inhibits expression of various target genes via binding gene promoters. BCL6 not only destroys interactions between T and B cells by $C D 80$ and PD-L1 but also inhibits $\mathrm{B}$ cell differentiation across decreasing expression of PRDM1 and IRF4 [32-37]. Above studies are consistent with the upregulation of $B C L 6$, and downregulation of other hub genes in our present study. PRDMI/BLIMPI encodes a transcriptional repressor, which is necessary for differentiation of B cells into plasma cells [38]. Studies reported that PRDM1 acts as a tumor suppressor gene in ABC-DLBCL in vivo mouse models [39, 40]. As previously described, PRDM1 is frequently inactivated by genetic alterations, including genetic deletions or mutations or transcriptional repression in ABC-DLBCL [38, 41]. Parekh et al. found that different genetic alterations within PRDM1 had adverse prognostic factors [42]. And inactivation of $P R D M 1$ can upregulate expression of $C-M Y C$ and downregulate expression of $p 53$ pathway molecule in ABC-DLBCL [42, 43]. These results suggest that inactivation of $P R D M 1$ is closely linked to development of ABC-DLBCL.

IRF4 was essential for regulating gene transcription and mitochondrial homeostasis in plasma cells [44]. IRF4 activates or is repressed by $B C L 6$, and co-expresses with PRPM1 affecting plasma cells development [45-47]. However, most studies found that it does not express PRDMI protein though the presence of IRF4 in ABC-DLBCL, suggesting other regulatory mechanisms between them [40]. Abnormal expression of IRF4 is linked to several blood malignancies. For example, expression of IRF4 is related 
to poor survival outcomes in peripheral T-cell lymphoma and chronic lymphocytic leukemia (CLL) $[48,49]$. What is more, studies showed IRF4 dysregulation is associated with resistance to immunomodulatory compounds in Waldenström's macroglobulinemia and myeloma [50, 51]. A previous study has reported that Ibrutinib downregulates IRF4 and consequently synergizes with lenalidomide in killing $A B C$ DLBCL [52]. Another study indicated mutation of IRF4 may explain the rel/ref phenotype of ABC-DLBCL [5]. Therefore, the role of IRF4 in ABC-DLBCL need further explore.

Lin et al. identified that upregulation of miR-30 family can directly downregulate $B C L 6$ in B-lymphocytes and lymphoma cells [53]. Current studies found miR-30 family played a significant role in various tumors. Zhang et al. proved miR-30d could inhibit autophagy thereby promoting cell apoptosis [54]. The higher expression of miRNA-30c had better outcome with tamoxifen treatment in breast cancer [55]. Another investigation found that overexpression of miR-30b and miR-30c have better outcome after TKIs treatment in non-small cell lung cancer [56]. Interestingly, miR-30 family is considered as oncogenic miRNA, too. For instance, Gaziel-Sovran et al. reported that miR-30b and miR-30d promoted invasion of melanoma cells leading to generated ILIO and reduced immune cells activation and recruitment [57]. Taken together, miR-30 family has complex functions in various cancers. However, the role of miR-30 family in Ibrutinib resistance of ABC-DLBCL has not been reported. Our study showed that miR-30 family may mediate Ibrutinib resistance in ABC-DLBCL, which is worthy of further exploration.

\section{Conclusion}

In summary, the present study has analyzed DEGs based on two microarray expression (GSE138126 and GSE93985). $B C L 6$ was identified as the core gene for Ibrutinib resistance in ABC-DLBCL. Using another expression profile (GSE93984) showed that BCL6 highly expressed in no responsive ABC-DLBCL patients after Ibrutinib treatment. Further study found that $B C L 6$ inhibitor may increase the sensitivity of C481S BTK HBL-1 cells to Ibrutinib therapy. And miRNA target prediction results showed that miR-30 family were involved in Ibrutinib resistance in ABC-DLBCL. And miR-30 family can directly downregulate $B C L 6$ which was reported before [53]. The BCL6 maybe a potential target overcoming Ibrutinib resistance in ABC-DLBCL.

Acknowledgements We thank Prof. Zhu for providing the C481S BTK HBL-1 cells
Author contributions JL, YH, and YZ contributed equally to this work and are co-first authors. JH and JL conceived and designed the study; YZ and YH found and analyzed the data, YC and JW carried experiments, LW and PJ wrote the paper, JH reviewed the paper.

Funding This work was supported by National Natural Science Foundation of China (81870135, 81470326, U2005204, 82000142), the Construction Project of the Fujian Medical Center of Hematology (Min201704), the Cooperation Project of University and Industry in Fujian Province (2017Y4005), Startup found of scientific research, Fujian medical university $(2019$ QH1022, 2019QH2012, 2019QH2016).

Availability of data and materials GSE138126, GSE93985, and GSE9394 were downloaded from the Gene Expression Omnibus (GEO) database.

\section{Compliance with ethical standards}

Conflict of interest There is no conflict of interest to disclose.

Consent to participate All authors agree to submit articles for publication.

Consent for publication All authors agree with publication.

Ethical approval The studies involving human participants were reviewed and approved by all data are from public database on the internet.

Open Access This article is licensed under a Creative Commons Attribution 4.0 International License, which permits use, sharing, adaptation, distribution and reproduction in any medium or format, as long as you give appropriate credit to the original author(s) and the source, provide a link to the Creative Commons licence, and indicate if changes were made. The images or other third party material in this article are included in the article's Creative Commons licence, unless indicated otherwise in a credit line to the material. If material is not included in the article's Creative Commons licence and your intended use is not permitted by statutory regulation or exceeds the permitted use, you will need to obtain permission directly from the copyright holder. To view a copy of this licence, visit http://creativecommons.org/licenses/by/4.0/.

\section{References}

1. Bachy E, Salles G. Treatment approach to newly diagnosed diffuse large B-cell lymphoma. Semin Hematol. 2015;52(2):107-18. https ://doi.org/10.1053/j.seminhematol.2015.01.001.

2. Swerdlow SH, Campo E, Pileri SA, Harris NL, Stein H, Siebert $\mathrm{R}$, et al. The 2016 revision of the World Health Organization classification of lymphoid neoplasms. Blood. 2016;127(20):2375-90. https://doi.org/10.1182/blood-2016-01-643569.

3. Lenz G, Wright G, Dave SS, Xiao W, Powell J, Zhao H. Stromal gene signatures in large-B-Cell Lymphomas. N Engl J Med. 2008;359(22):2313-23. https://doi.org/10.1056/NEJMoa0802885.

4. Li S, Young KH, Medeiros LJ. Diffuse large B-cell lymphoma. Pathology. 2018;50(1):74-87. https://doi.org/10.1016/j.patho 1.2017.09.006.

5. Mareschal S, Dubois S, Viailly PJ, Bertrand P, Bohers E, Maingonnat $\mathrm{C}$, et al. Whole exome sequencing of relapsed/refractory patients expands the repertoire of somatic mutations in 
diffuse large B-cell lymphoma. Genes Chromosomes Cancer. 2016;55(3):251-67. https://doi.org/10.1002/gcc.22328.

6. Morin RD, Assouline S, Alcaide M, Mohajeri A, Johnston RL, Chong L, et al. Genetic landscapes of relapsed and refractory diffuse large B-cell lymphomas. Clin Cancer Res. 2016;22(9):2290300. https://doi.org/10.1158/1078-0432.ccr-15-2123.

7. Jørgensen LK, Poulsen MØ, Laursen MB, Marques SC, Johnsen HE, Bøgsted M, et al. MicroRNAs as novel biomarkers in diffuse large B-cell lymphoma-a systematic review. Dan Med J. 2015;62(5):A5048.

8. Garzon R, Calin GA, Croce CM. MicroRNAs in cancer. Annu Rev Med. 2009;60:167-79. https://doi.org/10.1146/annur ev.med.59.053006.104707.

9. Ting CY, Liew SM, Price A, Gan GG, Bee-Lan Ong D, Tan SY, et al. Clinical significance of aberrant microRNAs expression in predicting disease relapse/refractoriness to treatment in diffuse large B-cell lymphoma: a meta-analysis. Crit Rev Oncol Hematol. 2019;144:102818. https://doi.org/10.1016/j.critrevonc .2019.102818.

10. Burger JA, Buggy JJ. Bruton tyrosine kinase inhibitor ibrutinib (PCI-32765). Leuk Lymphoma. 2013;54(11):2385-91. https://doi. org/10.3109/10428194.2013.777837.

11. Wilson WH, Young RM, Schmitz R, Yang Y, Pittaluga S, Wright $\mathrm{G}$, et al. Targeting B cell receptor signaling with ibrutinib in diffuse large B cell lymphoma. Nat Med. 2015;21(8):922-6. https:// doi.org/10.1038/nm.3884.

12. Edgar R, Domrachev M, Lash AE. Gene expression omnibus: NCBI gene expression and hybridization array data repository. Nucleic Acids Res. 2002;30(1):207-10. https://doi.org/10.1093/ nar/30.1.207.

13. Barrett T, Wilhite SE, Ledoux P, Evangelista C, Kim IF, Tomashevsky M, et al. NCBI GEO: archive for functional genomics data sets-update. Nucleic Acids Res. 2013;41:D991-5. https:// doi.org/10.1093/nar/gks1193.

14. da Huang W, Sherman BT, Lempicki RA. Systematic and integrative analysis of large gene lists using DAVID bioinformatics resources. Nat Protoc. 2009;4(1):44-57. https://doi.org/10.1038/ nprot.2008.211.

15. Szklarczyk D, Gable AL, Lyon D, Junge A, Wyder S, HuertaCepas J, et al. STRING v11: protein-protein association networks with increased coverage, supporting functional discovery in genome-wide experimental datasets. Nucleic Acids Res. 2019;47:D607-13. https://doi.org/10.1093/nar/gky1131.

16. Almeida D, Azevedo V, Silva A, Baumbach J. PetriScapea plugin for discrete Petri net simulations in Cytoscape. Journal of integrative bioinformatics. 2016;13(1):284. https://doi. org/10.2390/biecoll-jib-2016-284.

17. Gao J, Aksoy BA, Dogrusoz U, Dresdner G, Gross B, Sumer $\mathrm{SO}$, et al. Integrative analysis of complex cancer genomics and clinical profiles using the cBioPortal. Sci Signal. 2013. https://doi. org/10.1126/scisignal.2004088.

18. Wong N, Wang X. miRDB: an online resource for microRNA target prediction and functional annotations. Nucleic Acids Res. 2015;43:D146-52. https://doi.org/10.1093/nar/gku1104.

19. Sauter CS, Matasar MJ, Schoder H, Devlin SM, Drullinsky P, Gerecitano J, et al. A phase 1 study of ibrutinib in combination with R-ICE in patients with relapsed or primary refractory DLBCL. Blood. 2018;131(16):1805-8. https://doi.org/10.1182/ blood-2017-08-802561.

20. Younes A, Sehn LH, Johnson P, Zinzani PL, Hong X, Zhu J, et al. Randomized phase III trial of ibrutinib and rituximab plus cyclophosphamide, doxorubicin, vincristine, and prednisone in non-germinal center B-cell diffuse large B-cell lymphoma. J Clin Oncol. 2019;37(15):1285-95. https://doi.org/10.1200/ jco.18.02403.
21. Dreyling M, Jurczak W, Jerkeman M, Silva RS, Rusconi C, Trneny M, et al. Ibrutinib versus temsirolimus in patients with relapsed or refractory mantle-cell lymphoma: an international, randomised, open-label, phase 3 study. Lancet (London, England). 2016;387(10020):770-8. https://doi.org/10.1016/s0140 $-6736(15) 00667-4$.

22. Maddocks KJ, Ruppert AS, Lozanski G, Heerema NA, Zhao W, Abruzzo L, et al. Etiology of ibrutinib therapy discontinuation and outcomes in patients with chronic lymphocytic leukemia. JAMA Oncol. 2015;1(1):80-7. https://doi.org/10.1001/jamao ncol.2014.218.

23. Basso K, Dalla-Favera R. BCL6: master regulator of the germinal center reaction and key oncogene in B cell lymphomagenesis. Adv Immunol. 2010;105:193-210. https://doi.org/10.1016/s0065 -2776(10)05007-8.

24. Hatzi K, Melnick A. Breaking bad in the germinal center: how deregulation of BCL6 contributes to lymphomagenesis. Trends Mol Med. 2014;20(6):343-52. https://doi.org/10.1016/j.molme d.2014.03.001.

25. Lossos IS, Jones CD, Warnke R, Natkunam Y, Kaizer H, Zehnder $\mathrm{JL}$, et al. Expression of a single gene, BCL-6, strongly predicts survival in patients with diffuse large B-cell lymphoma. Blood. 2001;98(4):945-51. https://doi.org/10.1182/blood.v98.4.945.

26. Hurtz C, Chan LN, Geng H, Ballabio E, Xiao G, Deb G, et al. Rationale for targeting BCL6 in MLL-rearranged acute lymphoblastic leukemia. Genes Dev. 2019;33(17-18):1265-79. https:// doi.org/10.1101/gad.327593.119.

27. Eskandari S, Yazdanparast R. Bcl6 gene-silencing facilitates PMA-induced megakaryocyte differentiation in K562 cells. J Cell Commun Signal. 2017;11(4):357-67. https://doi.org/10.1007/ s12079-017-0395-5.

28. Duy C, Hurtz C, Shojaee S, Cerchietti L, Geng H, Swaminathan $\mathrm{S}$, et al. BCL6 enables $\mathrm{Ph}+$ acute lymphoblastic leukaemia cells to survive BCR-ABL1 kinase inhibition. Nature. 2011;473(7347):384-8. https://doi.org/10.1038/nature09883.

29. Wu HB, Lv WF, Wang YX, Li YY, Guo W. BCL6 promotes the methotrexate-resistance by upregulating ZEB1 expression in children with acute B lymphocytic leukemia. Eur Rev Med Pharmacol Sci. 2018;22(16):5240-7. https://doi.org/10.26355/eurrev_20180 8_15722.

30. Devin J, Kassambara A, Bruyer A, Moreaux J, Bret C. Phenotypic characterization of diffuse large B-cell lymphoma cells and prognostic impact. J Clin Med. 2019. https://doi.org/10.3390/jcm80 71074.

31. Cardenas MG, Yu W, Beguelin W, Teater MR, Geng H, Goldstein RL, et al. Rationally designed BCL6 inhibitors target activated B cell diffuse large B cell lymphoma. J Clin Invest. 2016;126(9):3351-62. https://doi.org/10.1172/jci85795.

32. Parekh S, Polo JM, Shaknovich R, Juszczynski P, Lev P, Ranuncolo SM, et al. BCL6 programs lymphoma cells for survival and differentiation through distinct biochemical mechanisms. Blood. 2007;110(6):2067-74. https://doi.org/10.1182/blood-2007-01069575.

33. Shaffer AL, Yu X, He YS, Boldrick J, Chan EP, Staudt LM. BCL-6 represses genes that function in lymphocyte differentiation, inflammation, and cell cycle control. Immunity. 2000;13:199-212. https://doi.org/10.1016/s1074-7613(00)00020-0.

34. Tunyaplin C, Shaffer AL, Angelin-Duclos CD, Yu X, Staudt LM, Calame KL. Direct repression of prdm1 by Bcl-6 inhibits plasmacytic differentiation. J Immunol (Baltimore, Md: 1950). 2004;173(2):1158-65. https://doi.org/10.4049/jimmu nol.173.2.1158.

35. Niu H, Cattoretti G, Dalla-Favera R. BCL6 controls the expression of the B7-1/CD80 costimulatory receptor in germinal center B cells. J Exp Med. 2003;198(2):211-21. https://doi.org/10.1084/ jem.20021395. 
36. Basso K, Saito M, Sumazin P, Margolin AA, Wang K, Lim WK, et al. Integrated biochemical and computational approach identifies BCL6 direct target genes controlling multiple pathways in normal germinal center B cells. Blood. 2010;115(5):975-84. https ://doi.org/10.1182/blood-2009-06-227017.

37. Basso K, Dalla-Favera R. Roles of BCL6 in normal and transformed germinal center B cells. Immunol Rev. 2012;247(1):17283. https://doi.org/10.1111/j.1600-065X.2012.01112.x.

38. Shapiro-Shelef M, Lin KI, McHeyzer-Williams LJ, Liao J, McHeyzer-Williams MG, Calame K. Blimp-1 is required for the formation of immunoglobulin secreting plasma cells and preplasma memory B cells. Immunity. 2003;19(4):607-20. https:// doi.org/10.1016/s1074-7613(03)00267-x.

39. Calado DP, Zhang B, Srinivasan L, Sasaki Y, Seagal J, Unitt C, et al. Constitutive canonical NF- $\kappa \mathrm{B}$ activation cooperates with disruption of BLIMP1 in the pathogenesis of activated B cell-like diffuse large cell lymphoma. Cancer Cell. 2010;18(6):580-9. https ://doi.org/10.1016/j.ccr.2010.11.024.

40. Mandelbaum J, Bhagat G, Tang H, Mo TW, Brahmachary M, Shen Q, et al. BLIMP1 is a tumor suppressor gene frequently disrupted in activated B cell-like diffuse large B cell lymphoma. Cancer Cell. 2010;18(6):568-79. https://doi.org/10.1016/j. ccr.2010.10.030.

41. Tam W, Gomez M, Chadburn A, Lee JW, Chan WC, Knowles DM. Mutational analysis of PRDM1 indicates a tumor-suppressor role in diffuse large B-cell lymphomas. Blood. 2006;107(10):4090100. https://doi.org/10.1182/blood-2005-09-3778.

42. Xia Y, Xu-Monette ZY, Tzankov A, Li X, Manyam GC, Murty V, et al. Loss of PRDM1/BLIMP-1 function contributes to poor prognosis of activated B-cell-like diffuse large B-cell lymphoma. Leukemia. 2017;31(3):625-36. https://doi.org/10.1038/leu.2016.243.

43. Zhang XY, Ma ZP, Cui WL, Pang XL, Chen R, Wang L, et al. Impact of PRDM1 gene inactivation on C-MYC regulation in diffuse large B-cell lymphoma. Chin J Pathol. 2018;47(1):25-31. https://doi.org/10.3760/cma.j.issn.0529-5807.2018.01.006.

44. Low MSY, Brodie EJ, Fedele PL, Liao Y, Grigoriadis G, Strasser A, et al. IRF4 activity is required in established plasma cells to regulate gene transcription and mitochondrial homeostasis. Cell Rep. 2019;29(9):2634-45 e5. https://doi.org/10.1016/j.celre p.2019.10.097.

45. Angelin-Duclos C, Cattoretti G, Lin KI, Calame K. Commitment of B lymphocytes to a plasma cell fate is associated with Blimp-1 expression in vivo. J Immunol (Baltimore, Md: 1950). 2000;165(10):5462-71. https://doi.org/10.4049/jimmu nol.165.10.5462.

46. Shapiro-Shelef M, Calame K. Regulation of plasma-cell development. Nat Rev Immunol. 2005;5(3):230-42. https://doi. org/10.1038/nri1572.

47. Klein U, Dalla-Favera R. Germinal centres: role in B-cell physiology and malignancy. Nat Rev Immunol. 2008;8(1):22-33. https:// doi.org/10.1038/nri2217.

48. Heo MH, Park HY, Ko YH, Kim WS, Kim SJ. IRF4/MUM1 expression is associated with poor survival outcomes in patients with peripheral T-cell lymphoma. J Cancer. 2017;8(6):1018-24. https://doi.org/10.7150/jca.17358.

49. Chang CC, Lorek J, Sabath DE, Li Y, Chitambar CR, Logan B, et al. Expression of MUM1/IRF4 correlates with clinical outcome in patients with B-cell chronic lymphocytic leukemia. Blood. 2002;100(13):4671-5. https://doi.org/10.1182/blood $-2002-01-0104$.

50. Zhu YX, Shi CX, Bruins LA, Wang X, Riggs DL, Porter B, et al. Identification of lenalidomide resistance pathways in myeloma and targeted resensitization using cereblon replacement, inhibition of STAT3 or targeting of IRF4. Blood Cancer J. 2019;9(2):19. https://doi.org/10.1038/s41408-019-0173-0.

51. Bertrand E, Jouy N, Manier S, Guillemette F, Guidez S, Eileen $\mathrm{B}$, et al. Role of IRF4 in resistance to immunomodulatory (IMid) compounds $®$ in Waldenström's macroglobulinemia. Onco Targets Ther. 2017;8(68):112917-27. https://doi.org/10.18632/oncotarget .22872 .

52. Yang Y, Shaffer AL III, Emre NT, et al. Exploiting synthetic lethality for the therapy of $\mathrm{ABC}$ diffuse large $\mathrm{B}$ cell lymphoma. Cancer Cell. 2012;21(6):723-37. https://doi.org/10.1016/j. ccr.2012.05.024.

53. Lin J, Lwin T, Zhao JJ, et al. Follicular dendritic cell-induced microRNA-mediated upregulation of PRDM1 and downregulation of BCL-6 in non-Hodgkin's B-cell lymphomas. Leukemia. 2011;251(1):145-52. https://doi.org/10.1038/leu.2010.230.

54. Zhang R, Xu J, Zhao J, Bai J. Mir-30d suppresses cell proliferation of colon cancer cells by inhibiting cell autophagy and promoting cell apoptosis. Tumour Biol. 2017;39(6):1010428317703984. https://doi.org/10.1177/1010428317703984.

55. Rodríguez-González FG, Sieuwerts AM, Smid M, Look MP, Meijer-van Gelder ME, de Weerd V, et al. MicroRNA-30c expression level is an independent predictor of clinical benefit of endocrine therapy in advanced estrogen receptor positive breast cancer. Breast Cancer Res Treat. 2011;127(1):43-51. https://doi. org/10.1007/s10549-010-0940-x.

56. Gu YF, Zhang H, Su D, Mo ML, Song P, Zhang F, et al. miR$30 \mathrm{~b}$ and miR-30c expression predicted response to tyrosine kinase inhibitors as first line treatment in non-small cell lung cancer. Chin Med J. 2013;126(23):4435-9.

57. Gaziel-Sovran A, Segura MF, Di Micco R, Collins MK, Hanniford D, Vega-Saenz de Miera E, et al. miR-30b/30d regulation of GalNAc transferases enhances invasion and immunosuppression during metastasis. Cancer Cell. 2011;20(1):104-18. https://doi. org/10.1016/j.ccr.2011.05.027.

Publisher's Note Springer Nature remains neutral with regard to jurisdictional claims in published maps and institutional affiliations. 